\title{
A combination of gefitinib and FOLFOX-4 as first-line treatment in advanced colorectal cancer patients. A GISCAD multicentre phase II study including a biological analysis of EGFR overexpression, amplification and NF-kB activation
}

\author{
S Cascinu ${ }^{*, I}$, R Berardi', S Salvagni ${ }^{2}$, GD Beretta ${ }^{3}$, V Catalano ${ }^{4}$, F Pucci ${ }^{2}$, A Sobrero ${ }^{5}$, P Tagliaferri ${ }^{6}$, \\ R Labianca ${ }^{3}$, M Scartozzi ${ }^{1}$, F Crocicchio ${ }^{\dagger, 7}$, E Mari $^{7}$ and A Ardizzoni ${ }^{2}$ \\ 'Department of Medical Oncology, Università Politecnica delle Marche, Ancona, Italy; ${ }^{2}$ Department of Medical Oncology, Azienda Ospedaliero- \\ Universitaria di Parma, Italy; ${ }^{3}$ Department of Medical Oncology, Azienda Ospedaliera di Bergamo, Bergamo, Italy; ${ }^{4}$ Department of Medical Oncology, \\ Azienda Ospedaliera S Salvatore, Pesaro, Italy; ${ }^{5}$ Department of Medical Oncology, Azienda Ospedaliera S Martino, Genova, Italy; ${ }^{6}$ Department of \\ Medical Oncology, Università della Magna Grecia, Catanzaro, Italy; ${ }^{7}$ Research and Development, AstraZeneca, Basiglio, Italy
}

Interesting activity has been reported by combining chemotherapy with cetuximab. An alternative approach for blocking EGFR function has been the development of small-molecule inhibitors of tyrosine kinase domain such as gefitinib. We designed a multicentre phase II study in advanced colorectal cancer combining gefitinib + FOLFOX in order to determine the activity and to relate EGFR expression and gene amplification and NF-kB activation to therapeutic results. Patients received FOLFOX-4 regimen plus gefitinib as first-line treatment. Tumour samples were analysed for EGFR protein expression by immunohistochemical analysis and for EGFR gene amplification by fluorescence in situ hybridisation (FISH), chromogenic in situ hybridisation (CISH) and NF-kB activation. Forty-three patients were enrolled into this study; 15 patients experienced a partial response (response rate $=34.9 \%$ ), whereas other I2 (27.9\%) had a stable disease. Median progression-free survival (PFS) was 7.8 months and median overall survival (OS) was I3.9 months. We did not find any relationship with EGFR overexpression, gene amplification, while NF-kB activation was associated with a resistance to therapy. Gefitinib does not seem to increase the activity of FOLFOX in advanced colorectal cancer even in patients overexpressing EGFR or with EGFR amplification. Furthermore, while NF-kB activation seems to predict resistance to chemotherapy as demonstrated 'in vitro' models, gefitinib does not overcome this mechanism of resistance, as reported for cetuximab. British Journal of Cancer (2008) 98, 7 I-76. doi:10.1038/sj.bjc.6604I2I www.bjcancer.com

Published online 4 December 2007

(c) 2008 Cancer Research UK

Keywords: colorectal cancer; gefitinib; NF-kB

FOLFOX is a generally recognised first-line chemotherapy for metastatic colorectal cancer. Patients receiving this combination chemotherapy achieved a $50 \%$ response rate, with a time to progression (TTP) of 9 months and an overall survival of 14 months (de Gramont et al, 2000; Goldberg et al, 2004; Colucci et al, 2005; Meyerhardt and Mayer, 2005).

Recently, interesting activity has been reported by combining FOLFOX or FOLFIRI with EGFR inhibitors. Epidermal growth factor receptor has been demonstrated to be overexpressed in about $70-80 \%$ of colorectal tumours and its overexpression is associated with a worse prognosis (Laskin and Sandler, 2004).

Epidermal growth factor receptor inhibition may be obtained by blocking the extracellular part of the receptor (Ellis and Hoff, 2004). Cetuximab, a monoclonal antibody, has been demonstrated to produce a $10 \%$ response rate in monotherapy and a $20 \%$ when

*Correspondence: Professor S Cascinu; E-mail: cascinu@yahoo.com Received 23 August 2007; revised I November 2007; accepted 2 November 2007; published online 4 December 2007 combined with irinotecan even in patients refractory to this drug (Cunningham et al, 2004; Lenz et al, 2006).

An alternative approach for blocking EGFR function in cancer cells has been the development of small molecules able to interfere with the enzymatic activity of the ligand-activated EGFR (Ellis and Hoff, 2004). Gefitinib is a potent small-molecule inhibitor of tyrosine kinase domain of EGFR. It has demonstrated activity in non-small-cell lung cancer (Cohen et al, 2004). It is orally available and is an attractive therapeutic option in colorectal cancer patients. In fact, preclinical data demonstrated a synergism between gefitinib and oxaliplatin and thymidilate synthetase inhibitors (Ciardiello et al, 2000; Xu et al, 2003; Van Schaeybroeck et al, 2005).

Phase I studies showed the feasibility and the safety of the combination of gefitinib with FOLFOX (Kindler et al, 2005).

Based on these premises, we initiated a multicentre phase II study in advanced colorectal cancer, combining gefitinib with FOLFOX in order to determine the activity of such a combination and to relate EGFR expression and gene amplification to therapeutic results, as well as if resistance to chemotherapy 
induced by NF-kB activation may be overcome by gefitinib, as it happens for cetuximab.

\section{PATIENTS AND METHODS}

Patients were considered eligible for this study if they were older than 18 years of age and had histologically confirmed metastatic colorectal adenocarcinoma. Patients were not allowed to receive any kind of prior treatment for their metastatic disease. Other eligibility criteria included measurable disease by Response Evaluation Criteria in Solid Tumors Group (RECIST) criteria, no prior exposition to EGFR inhibitors, an Eastern Cooperative Oncology Group (ECOG) performance status $0-1$, adequate blood, renal and liver function. Analysis of tumour EGFR status was not required for inclusion in this study. The treatment protocol was approved by local Ethical Committees. This study was sponsored by AstraZeneca (study number 1839IL/0119).

\section{Treatment}

Initially a cohort of five patients received standard doses of FOLFOX-6 with oxaliplatin at the dose of $100 \mathrm{mg} \mathrm{m}^{-2}$ in combination with $250 \mathrm{mg}$ of gefitinib daily. After five patients were treated initially, we decided to expand the cohort to 42 patients, administering FOLFOX-4 at dosages previously published (oxaliplatin $85 \mathrm{mg} \mathrm{m}^{-2}$ ) (Andre et al, 1999) due to a better profile of toxicity and tolerability.

Gefitinib at the dose of $250 \mathrm{mg}$ has been taken once a day, every day about the same time. It could be taken with or without food. If the patients forgot to take a dose, they took the last missed dose as soon as they remembered, as long as it was at least $12 \mathrm{~h}$ before the next dose is due.

All toxicities were graded according to the NCI-CTC version 2.0. Retreatment at the start of each cycle required adequate haematologic function and resolution of all toxicities of CTC grade 2 or more.

Treatment was continued until development of progressive disease or unacceptable toxicity, withdrawal of patients consent or decision to perform surgical resection of disease.

\section{Evaluation}

Baseline tumour measurements by computed tomography were obtained within 28 days before study treatment was started. Physical examination, including medical history, laboratory studies and assessment of performance status, were conducted at the beginning of each 2-week cycle. Patients were asked to keep a diary of daily gefitinib ingestion and record their experience of nausea and diarrhoea.

Tumour response was evaluated approximately every 8 weeks by computed tomography imaging and tumour measurement performed using RECIST criteria.

\section{Statistical analysis}

Fleming's method was used to calculate the number of patients required (O'Brien and Fleming, 1979).

A sample size of 42 patients is sufficient to give an $80 \%$ probability of rejecting a baseline response rate of $50 \%$ with an exact $5 \%$ one-sided significance test when the true response is at the clinically relevant rate of $70 \%$. The hypothesis that the response rate is equal or less than the baseline is rejected if 27 or more responses are observed out of the 42 patients. Using the methods of A'Hearn (2004), the exact size and power of this test are 4.4 and $83.6 \%$, respectively.

The baseline response rate used for the null hypothesis has been set at $50 \%$. Rejecting the null hypothesis indicates that the activity of the combination is at least similar to that observed using the FOLFOX regimen alone, and therefore that the addition of gefitinib does not compromise the activity of FOLFOX. In the absence of gefitinib, the proposed FOLFOX regimen produces response rates ranging of approximately $50 \%$ in the first-line setting, with median progression free survival of 9 months. The addition of gefitinib to FOLFOX is expected to increase the response rate to above $65 \%$. However, the FOLFOX regimen is associated with toxicity, with grade 3-4 neutropaenia observed in $42 \%$ of patients, grade $3-4$ diarrhoea and grade 3 neurosensory toxicity in $18 \%$. In this study there is a potential for overlapping toxicity, and therefore a higher baseline response rate has not been used to limit the number of patients exposed to the treatment before obtaining further data on safety and some preliminary information on activity.

\section{Immunohistochemical analysis and gene amplification}

Tumour samples, formalin-fixed and paraffin-included, were analysed for EGFR protein expression by immunohistochemical analysis and for EGFR gene amplification by fluorescence in situ hybridisation (FISH) and chromogenic in situ hybridisation (CISH).

Immunohistochemistry The immunohistochemical study was performed and graded using kit EGFR PharmaDx ${ }^{\mathrm{TM}}$ (DakoCytomation, Carpinteria, CA, USA) according to the manufacturer's instructions as previously published (Scartozzi et al, 2004).

The EGFR-positive immunostained areas were identified for creation of the section treated with FISH and CISH technique; in addition areas at random were selected in negative tumour for EGFR immunostain.

\section{FISH analysis of EGFR gene copy number}

Analysis of EGFR amplification was performed by using the standard with the dual-colour EGFR Spectrum Orange ${ }^{\mathrm{TM}} / \mathrm{CEP}^{\circledR}{ }^{\mathbb{R}}$ Spectrum Green ${ }^{\mathrm{TM}}$ probe (Visys, Downers Grove, IL USA). In brief, paraffin sections were deparaffinised, dehydrated in ethanol and air-dried. After treatment in $0.05 \%$ pepsin $/ 0.1 \mathrm{~N} \mathrm{HCl}$ for $45 \mathrm{~min}$ at $37^{\circ} \mathrm{C}$, the sample were aged in $0.1 \% \mathrm{NP}-40 / 2 \times \mathrm{SSC}$ (standard saline citrate solution) for $10 \mathrm{~min}$ at $37^{\circ} \mathrm{C}$ and their DNA was denatured by treatment in $70 \%$ formamide $2 \times$ SSC for $4 \mathrm{~min}$ at $85^{\circ} \mathrm{C}$. A measure of $5 \mu \mathrm{l}$ of the probe solution was then placed on a glass slide with a coverslip. The sample slides were hybridised overnight at $37^{\circ} \mathrm{C}$ and washed, before in $0.4 \times \mathrm{SSC} / 0.3 \% \mathrm{NP}-40$ at $73^{\circ} \mathrm{C}$ for $2 \mathrm{~min}$. Nuclei were counterstained with $4^{\prime}, 6$-diamino2-phenylindole dihydrochloride and $p$-phenylenediamine in phosphate-buffered saline (PBS) and glycerol (DAPI II) (Vysis, Downers Grove, IL, USA). Each FISH assay included normal breast tissue sections, as negative control, and sections of breast cancer previously confirmed to have amplification of EGFR as positive control.

Analyses were performed using a fluorescence microscope (Nikon Optphot-2 and Quips Genetic Workstation) equipped with the Tripple Bandpass Filter set (Vysis) for DAPI II, SpectrumOrange and SpectrumGreen and Filter sets specific to SpectrumOrange and SpectrumGreen.

Only individual and well-delineated cells were scored; overlapping cells were excluded from the analysis. At least 60 cells were scored for each case and control sample.

Each tumour was assessed by the average and the maximum numbers of the copies of EGFR gene per cell, and the average EGFR/chromosome 7 copy number (CEP7) ratio.

Amplification was defined as ratio of EGFR signals to chromosome 7 centromere signals of 2 or more. 


\section{CISH analysis of EGFR gene copy number}

Chromogenic in situ hybridisation for the EGFR gene was performed according to the manufacturer's instructions (Zymed Laboratories Inc., South San Francisco, CA, USA).

Briefly, the sections of the formalin-fixed and paraffinembedded tissue were incubated at $55^{\circ} \mathrm{C}$ overnight. The slides were deparaffinised in xylene and graded ethanols; heat pretreatment was carried out in the pretreatment buffer (Zymed Laboratories Inc.) at $96^{\circ} \mathrm{C}$ for $15 \mathrm{~min}$.

The tissue was digested with pepsin for $10 \mathrm{~min}$ at room temperature, successively was washed with deionised water, dehydrated with graded ethanol and air-dried.

After application of Zymed Spot-Light ${ }^{\circledR}$ oligoxigenin labelled EGFR probe (Zymed Laboratories Inc.), the slides were coverslipped and edges sealed with rubber cement. The slides were heated at $92^{\circ} \mathrm{C}$ for $5 \mathrm{~min}$, followed by overnight incubation at $37^{\circ} \mathrm{C}$ using moisturised chamber.

Post-hybridisation wash was performed the next day, followed by immunodetection using the $\mathrm{CISH}^{\mathrm{TM}}$ polymer detection kit (Zymed Laboratories Inc.).

The CISH signals were seen as dark brown dots and counted in at last 100 nuclei with a light microscope using $\times 40$ objective; only individual and well-delineated cells were scored, and overlapping cells were excluded from the analysis. Also the average gene copies per nucleus for each tissue sections were calculated.

\section{NF-kB}

Nuclear factor-kB was evaluated with an immunohistochemical technique on 3- to 5- $\mu$ m-thick tissue sections obtained from paraffin-embedded specimens fixed in $10 \%(\mathrm{v} / \mathrm{v})$ neutral buffered formalin.

The sections were deparaffinised in xylene, rehydrated in graded ethanol, washed in PBS and heated in microwave at $98^{\circ} \mathrm{C}$, with EDTA buffer (1 mM; pH 8).

Peroxide blocking was performed with $0.3 \% \mathrm{H}_{2} \mathrm{O}$ in methanol at room temperature for $30 \mathrm{~min}$.

The mouse monoclonal antibody raised against amino acids 1 286 of NF-kB p65 of human origin was used (1:150 dilution; Santa Cruz Biotechnology, Inc, Santa Cruz, CA, USA) and incubated for $1 \mathrm{~h}$ at room temperature.

Incubation with the secondary antibody (EnVision System ${ }^{\mathbb{R}}$; DakoCytomation, Carpinteria, CA, USA/HRP) was performed for $30 \mathrm{~min}$, followed by application of diaminobenzidine chromogen for $5 \mathrm{~min}$. Subsequently, the slides were counterstained with Meyer's haematoxylin for $1 \mathrm{~min}$, dehydrated in a graded series of alcohol, treated with xylene and coverslipped.

The slides were evaluated by light microscopy independently by two pathologists.

Nuclear factor-kB expression was detected as nuclear and cytoplasmic brown staining of neoplastic cell, with various intensity. Positivity of the tumour for NF-kB expression was defined as only distinct nuclear immunostaining, which is considered as activated NF-kB, and is quantified by a percentage score (range $0-100$ ).

The lymphocytes within the tissue sections were used as positive internal controls, which showed positive nuclear staining in all runs.

The negative control was used during optimisation of the method and did not show any staining.

\section{RESULTS}

\section{Baseline characteristics}

Between October 2002 and September 2004, 43 patients were enrolled into this study. Although four patients discontinued chemotherapy before completion of two cycles and were lost to follow-up, all 43 patients enrolled are included in the toxicity and efficacy analyses, on the basis of intention-to-treat.

The baseline characteristics of the 43 patients are listed in Table 1.

\section{Treatment administration}

The mean duration of trial therapy was 26.07 weeks (range $0.14-83.43$ weeks).

\section{Activity}

The primary activity end point for this study was objective response rate. By intent-to-treat analysis, 15 patients experienced a partial response (response rate $=34.9 \%$ ), whereas other 12 patients (27.9\%) had a stable disease for at least four cycles (Table 2). The secondary efficacy end points were progression-free survival (PFS) and overall survival (OS). The median PFS was 7.8 months $(95 \%$ CI: 6.7-10 months) (Figure 1). The median OS for all the patients was 13.9 months (95\% CI: 11.2-19.9 months) (Figure 2).

\section{Toxicities}

The toxicities of the study regimen represented a secondary end point. Thirty-five out of 43 patients (81.4\%) expressed some types of grade 3 or 4 toxicities. Neutropaenia and diarrhoea were the most common side effects. Additional grade 3-4 toxicities included transaminase increase (three patients: in one patient related to gefitinib, in one patient related to FOLFOX and in one

Table I Baseline characteristics of patients

\begin{tabular}{lr}
\hline Characteristics & $\boldsymbol{n}$ \\
\hline No. of patients & 43 \\
Median age, years (range) & $60(35-75)$ \\
Sex & \\
Male & $23(53.5 \%)$ \\
Female & $20(46.5 \%)$ \\
Performance status & \\
0 & $30(69.8 \%)$ \\
I & $13(30.2 \%)$ \\
Received prior adjuvant therapy & \\
Yes & $9(20.9 \%)$ \\
No & $34(79.1 \%)$ \\
Metastatic sites & \\
I site & \\
$>$ I site & $21(48.8 \%)$ \\
\hline
\end{tabular}

Table 2 Activity of the treatment

\begin{tabular}{lc}
\hline Parameter & $\boldsymbol{n}(\%)$ \\
\hline Partial response & $15 / 43(34.9 \%)$ \\
Stable disease & $12 / 43(27.9 \%)$ \\
Progressive disease & $12 / 43(27.9 \%)$ \\
& \\
Median response duration & 5.3 months (range: $0.9-20.9)$ \\
Median PFS & 7.8 months (95\% Cl: $6.7-10)$ \\
Median OS & 13.9 months (95\% Cl: $11.2-19.9)$ \\
\hline
\end{tabular}

$\mathrm{Cl}=$ confidence interval; $\mathrm{OS}=$ overall survival; $\mathrm{PFS}=$ progression-free survival. 


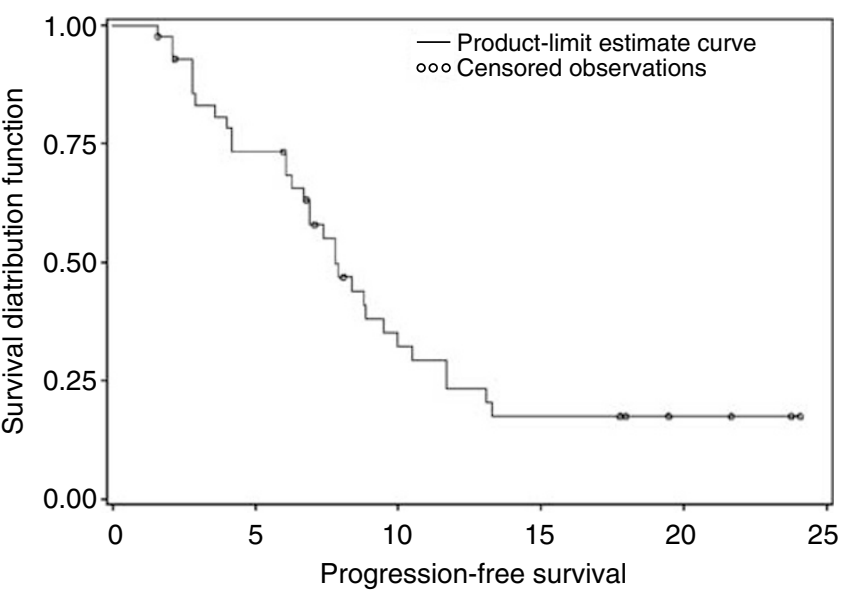

Figure I Kaplan-Meier plots for progression free survival.

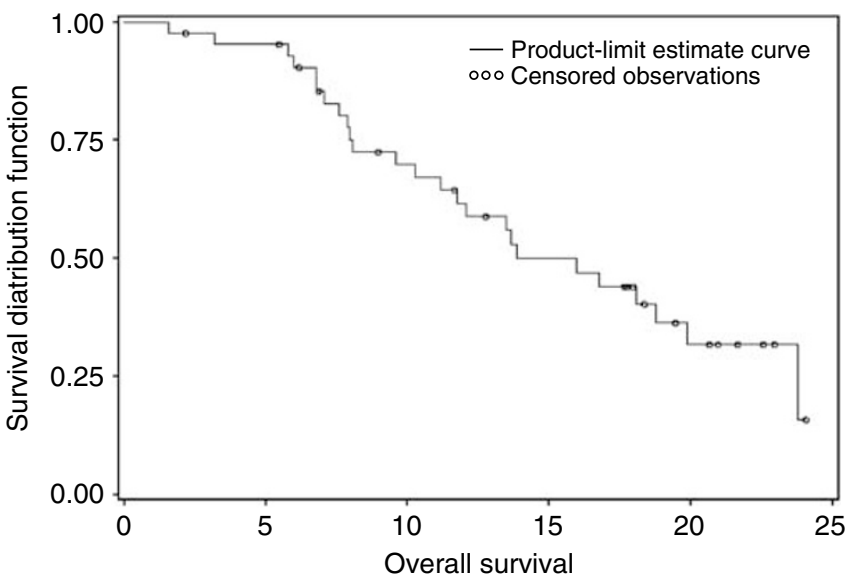

Figure 2 Kaplan-Meier plots for overall survival.

Table 3 Grade 3-4 toxicities (all causes)

\begin{tabular}{lcc}
\hline Toxicity & All grades (\%) & Grades 3-4 (\%) \\
\hline Neutropaenia & $26(60)$ & $18(42)$ \\
Thrombocytopaenia & $14(33)$ & - \\
Diarrhoea & $28(65)$ & $13(30)$ \\
Mucositis & $10(23)$ & $2(5)$ \\
Leukopaenia & $9(21)$ & $3(7)$ \\
Transaminase increase & $8(19)$ & $3(7)$ \\
Neuropathy & $11(25)$ & $1(2.3)$ \\
Dermatitis & $16(37)$ & $1(2.3)$ \\
\hline
\end{tabular}

patient related to both gefitinib and FOLFOX), leukopaenia related to FOLFOX (three patients), mucositis (two patients: in one patient related to FOLFOX and in one patient related to both gefitinib and FOLFOX) dermatitis or dry skin attributable to gefitinib in one patient and grade 3 peripheral neuropathy attributable to oxaliplatin in one patient. The most common toxicities for all causes are listed in Table 3.

\section{Immunoistochemistry analysis and gene amplification}

Results of EGFR gene analysis by FISH and CISH and NF-kB expression related to clinical response are summarised in Table 4. Data on 20 patients were available for biological analysis. Nuclear
Table 4 Available biological data on 20 patients

\begin{tabular}{|c|c|c|c|}
\hline \multicolumn{4}{|c|}{ NF-kB activation } \\
\hline & Yes (4) & No (16) & \\
\hline PR & 0 & 10 & $P<0.05$ \\
\hline SD & - & 5 & $P<0.05$ \\
\hline PD & 4 & 1 & $P<0.05$ \\
\hline \multicolumn{4}{|c|}{ EGFR overexpression } \\
\hline & Yes $(||)$ & No (9) & \\
\hline PR & 6 & 4 & $P=N S$ \\
\hline SD & 1 & 4 & $P=N S$ \\
\hline PD & 4 & । & $P=N S$ \\
\hline \multicolumn{4}{|c|}{ EGFR amplification } \\
\hline & Yes (3) & No (17) & \\
\hline PR & 1 & 9 & \\
\hline SD & 2 & 3 & \\
\hline PD & - & 5 & \\
\hline
\end{tabular}

$\mathrm{EGFR}=$ epidermal growth factor receptor; NF-kB $=$ nuclear factor-kB; NS $=$ nonsignificant; $P R=$ partial response; $P D=$ progressive disease; $S D=$ stable disease.

factor-kB was activated in four out of five patients who progressed, while it was not activated in all the 10 patients who achieved a PR. The results were statistically significant $(P<0.05)$.

Epidermal growth factor receptor was overexpressed in four out of the five patients who progressed, and in six of the 10 patients who had a partial response, but the results were not statistical significant.

Epidermal growth factor receptor was not amplificated in nine out of the 10 patients who achieved a partial response, and in all the five patients who progressed.

\section{DISCUSSION}

The number of available chemotherapy regimens against metastatic colon cancer is rapidly growing. Oxaliplatin or irinotecan in combination with infusional 5FU has been shown to be effective in achieving an improved response and TTP compared with 5FU/LV in colorectal cancer (Grothey et al, 2004).

The high level of EGFR response in colorectal cancer specimens has sparked great interest in using this target to develop more direct and specific therapies (Baselga and Arteaga, 2005). To date, positive results with EGFR inhibitors have only been reported for the monoclonal antibody cetuximab. Small-molecule inhibitors of EGFR have recently been tested in combination with chemotherapy in second-line treatment in colorectal cancer. Both gefitinib and erlotinib in combination with FOLFOX or capecitabine and oxaliplatin showed interesting results obtaining higher response rates than those expected with chemotherapy alone. In spite of the lack of benefit for the addition of gefitinib or erlotinib to standard chemotherapy in non-small-cell lung cancer, the results obtained in second-line therapy of colorectal cancer are a strong rationale to move this combination to first line treatment (Kuo et al, 2005; Meyerhardt et al, 2006).

In this multi-institutional phase II study of the combination of FOLFOX with gefitinib for previously untreated colorectal cancer patients, we observed a response rate of $34.9 \%$ and a median PFS of 7.8 months. The median survival for this population of patients was 13.9 months.

Our findings do not compare favourably with similar regimens in first line treatment. In fact, regimens with infusional 5FU and oxaliplatin result in similar response rates and TTP, suggesting that adding gefitinib to FOLFOX does not improve the efficacy of this regimen. These negative results may be due to a lack of efficacy of small molecules inhibitors of EGFR in combination with 
chemotherapy as in colorectal cancer or to the lack of patient selection (Giaccone et al, 2004; Herbst et al, 2004; Messersmith et al, 2004).

A recent study by Zampino and co-workers also evaluated the combination FOLFOX- 6 and gefitinib in a phase II trial including 56 patients. The results showed a higher response (complete response $=5.36 \%$; partial response $=66.1 \%$ ) and a higher disease control $(91.1 \% ; 95 \% \mathrm{CI}=80.4-97 \%)$. Due to the rather small numbers of patients and to the nature of the trial (phase II), in our opinion the differences between the above mentioned and our results are likely due to chance. Nevertheless, a comment could be addressed: in the trial of Zampino and co-workers, patients were enrolled if they had at least $20 \%$ cancer cells positive for EGFR, while in our trial only $1 \%$ of EGFR positivity was required as per standard practice (Zampino et al, 2007).

No biological correlative studies were able to demonstrate a predictive factor for cetuximab therapy activity. Nevertheless, we analysed EGFR overexpression and amplification and NF-kB activation in 20 out of 43 patients receiving gefitinib and chemotherapy. Table 4 summarises these findings. While EGFR overexpression determined by immunohistochemistry is not able to predict response to cetuximab, EGFR amplification was reported to be a good indicator of sensitivity to cetuximab in colorectal cancer. Nuclear factor-kB activation results in an increased resistance to chemotherapy, and the administration of an EGFR inhibitor seems to be able to revert this resistance (Sclabas et al, 2003).

In our study, we did not find any relationship between EGFR overexpression and response. In spite of the experience of Moroni et al (2005) where about $30 \%$ of tumours presented an EGFR amplification, we did not find it in any of our patients.

Similar data were reported by Garufi et al (2006) in 70 colorectal cancer patients. In fact, amplification was found in three patients only. Furthermore, Lenz et al (2006) reported that EGFR amplification is not related to response to cetuximab, questioning the role of EGFR amplification in the prediction of clinical activity of EGFR inhibitors.

The NF-kB transcriptional factor is constitutively activated in several tumours included colorectal cancer. Furthermore, it is activated by chemotherapy and it represents one of the most important mechanism of cell survival in response to chemotherapy resulting in resistance to treatment (Lind et al, 2001; Rakitinat et al, 2003; Sclabas et al, 2003; Guo et al, 2004; Voboril et al, 2004; Karin, 2006). In our study, activation of NF-kB was present in four cases. These patients did not respond to treatment, while all the patients with no NF-kB activation presented an objective response. This factor seems to be able to predict resistance to a treatment with FOLFOX and the addition of gefitinib does not overcome this resistance, as some preclinical data suggested for cetuximab (Sclabas et al, 2003). Our data are too limited to draw any definitive conclusion; nevertheless in our experience gefitinib does not increase the activity of FOLFOX combination in advanced colorectal cancer even in patients overexpressing EGFR or with EGFR amplification. Furthermore, while NF-kB activation seems to predict resistance to chemotherapy as demonstrated in 'in vitro' models, in our trial, although the small numbers, gefitinib does not overcome this mechanism of resistance as reported for cetuximab.

\section{REFERENCES}

A'Hearn B (2004) A restricted maximum likelihood estimator for truncated height samples. Econ Hum Biol 2(1): 5-19

Andre T, Bensmaine MA, Louvet C, Francois E, Lucas V, Desseigne F, Beerblock K, Bouche O, Carola E, Merrouche Y, Morvan F, DupontAndre G, de Gramont A (1999) Multicenter phase II study of bimonthly high-dose leucovorin, fluorouracil infusion, and oxaliplatin for metastatic colorectal cancer resistant to the same leucovorin and fluorouracil regimen. J Clin Oncol 17(11): 3560-3568

Baselga J, Arteaga CL (2005) Critical update and emergine trends in epidermal growth factor receptor targeting in cancer. J Clin Oncol 23: $2445-2459$

Ciardiello F, Caputo R, Bianco R, Damiano V, Pomatico G, De Placido S, Bianco AR, Tortora G (2000) Antitumor effect and potentiation of cytotoxic drugs activity in human cancer cells by ZD-1839 (Iressa), an epidermal growth factor receptor-selective tyrosine kinase inhibitor. Clin Cancer Res 6: 2053-2063

Cohen MH, Williams GA, Sridhara R, Chen G, McGuinn Jr WD, Morse D, Abraham S, Rahman A, Liang C, Lostritto R, Baird A, Pazdur R (2004) United States Food and Drug Administration drug approval summary: gefitinib (ZD1839; Iressa) tablets. Clin Cancer Res 10: 1212-1218

Colucci G, Gebbia V, Paoletti G, Giuliani F, Caruso M, Gebbia N, Carteni G, Agostara B, Pezzella G, Manzione L, Borsellino N, Misino A, Romito S, Durini E, Cordio S, Di Seri M, Lopez M, Maiello E, Montemurro S, Cramarossa A, Lorusso V, Di Bisceglie M, Chiarenza M, Valerio MR, Guida T, Leonardi V, Pisconti S, Rosati G, Carrozza F, Nettis G, Valdesi M, Filippelli G, Fortunato S, Mancarella S, Brunetti C (2005) Phase III randomized trial of FOLFIRI $v s$ FOLFOX4 in the treatment of advanced colorectal cancer: a multicenter study of the Gruppo Oncologico dell'Italia Meridionale. J Clin Oncol 23: 4866-4875

Cunningham D, Humblet Y, Siena S, Khayat D, Bleiberg H, Santoro A, Bets D, Mueser M, Harstrick A, Verslype C, Chau I, Van Cutsem E (2004) Cetuximab monotherapy and cetuximab plus irinotecan in irinotecanrefractory metastatic colorectal cancer. $N$ Engl J Med 351: $337-345$

de Gramont A, Figer A, Seymour M, Homerin M, Hmissi A, Cassidy J, Boni C, Cortes-Funes H, Cervantes A, Freyer G, Papamichael D, Le Bail N, Louvet C, Hendler D, de Braud F, Wilson C, Morvan F, Bonetti A (2000) Leucovorin and fluorouracil with or without oxaliplatin as first-line treatment in advanced colorectal cancer. J Clin Oncol 18(16): 2938-2947
Ellis LM, Hoff PM (2004) Targeting the epidermal growth factor receptor: an important incremental step in the battle against colorectal cancer. J Clin Oncol 22: $1177-1179$

Garufi C, Mottolese M, Cianciulli A, Zeuli M, Buglioni S, Torsello A, Vanni B, Campanella C, Merola R, Terzoli E (2006) Epidermal growth factor gene amplification is not frequent and cannot account for antitumor activity of cetuximab plus chemotherapy in advanced colorectal cancer patients. Proc Am Soc Clin Oncol 161: 3561

Giaccone G, Herbst RS, Manegold C, Scagliotti G, Rosell R, Miller V, Natale RB, Schiller JH, Von Pawel J, Pluzanska A, Gatzemeier U, Grous J, Ochs JS, Averbuch SD, Wolf MK, Rennie P, Fandi A, Johnson DH (2004) Gefitinib in combination with gemcitabine and cisplatin in advanced non-small-cell lung cancer: a phase III trial - INTACT 1. J Clin Oncol 22(5): $777-784$

Goldberg RM, Sargent DJ, Morton RF, Fuchs CS, Ramanathan RK, Williamson SK, Findlay BP, Pitot HC, Alberts SR (2004) A randomized controlled trial of fluorouracil plus leucovorin, irinotecan, and oxaliplatin combinations in patients with previously untreated metastatic colorectal cancer. J Clin Oncol 22: $23-30$

Grothey A, Sargent D, Goldberg RM, Schmoll HJ (2004) Survival of patients with advanced colorectal cancer improves with the availability of fluorouracil-leucovorin, irinotecan, and oxaliplatin in the course of treatment. J Clin Oncol 22: 1209-1214

Guo J, Verma UN, Gaynor RB, Frenkel EP, Becerra CR (2004) Enhanced chemosensitivity to irinotecan by RNA interference-mediated downregulation of the nuclear factor-kB p65 subunit. Clin Cancer Res 10: $3333-3341$

Herbst RS, Giaccone G, Schiller JH, Natale RB, Miller V, Manegold C, Scagliotti G, Rosell R, Oliff I, Reeves JA, Wolf MK, Krebs AD, Averbuch SD, Ochs JS, Grous J, Fandi A, Johnson DH (2004) Gefitinib in combination with paclitaxel and carboplatin in advanced nonsmall-cell lung cancer: a phase III trial - INTACT 2. J Clin Oncol 22(5): $785-794$

Karin M (2006) Nuclear factor-kB in cancer development and progression. Nature 441: $431-436$

Kindler HL, Friberg G, Skoog L, Wade-Oliver K, Vokes EE (2005) Phase I/II trial of gefitinib and oxaliplatin in patients with advanced colorectal cancer. Am J Clin Oncol 28: $340-344$ 
Kuo T, Cho CD, Halsey J, Wakelee HA, Advani RH, Ford JM, Fisher GA, Sikic BI (2005) Phase II study of gefitinib, fluorouracil, leucovorin and oxaliplatin therapy in previously treated patients with metastatic colorectal cancer. J Clin Oncol 23: 5613-5619

Laskin JJ, Sandler AB (2004) Epidermal growth factor receptor: a promising target in solid tumours. Cancer Treat Rev 30: 1-17

Lenz HJ, Van Cutsem E, Khambata-Ford S, Mayer RJ, Gold P, Stella P, Mirtsching B, Cohn AL, Pippas AW, Azarnia N, Tsuchihashi Z, Mauro DJ, Rowinsky EK (2006) Multicenter phase II and translational study of cetuximab in metastatic colorectal carcinoma refractory to irinotecan, oxaliplatin and fluoropyrimidines. J Clin Oncol 24: 4914-4921

Lind DS, Hochwald SN, Malaty J, Rekkas S, Hebig P, Mishra G, Moldawer LL, Copeland III EM, Mackay S (2001) Nuclear factor-kB is upregulated in colorectal cancer. Surgery 130(2): 363-369

Messersmith WA, Laheru DA, Senzer NN, Donehower RC, Grouleff P, Rogers T, Kelley SK, Ramies DA, Lum BL, Hidalgo M (2004) Phase I tria of irinotecan, infusional 5-fluorouracil, and leucovorin (FOLFIRI) with erlotinib (OSI-774): early termination due to increased toxicities. Clin Cancer Res 10: 6522-6527

Meyerhardt J, Mayer RJ (2005) Systemic therapy for colorectal cancer. $N$ Engl J Med 352: 476-487

Meyerhardt JA, Zhu AX, Enzinger PC, Ryan DP, Clark JW, Kulke MH, Earle CC, Vincitore M, Michelini A, Sheehan S, Fuchs CS (2006) Phase II study of capecitabine, oxaliplatin and erlotinib in previously treated patients with metastatic colorectal cancer. J Clin Oncol 24: 1892-1897

Moroni M, Veronese S, Benvenuti S, Marrapese G, Sartore-Bianchi A, Di Nicolantonio F, Gambacorta M, Siena S, Bardelli A (2005) Gene copy number for epidermal growth factor receptor (EGFR) and clinical response to antiEGFR treatment in colorectal cancer: a cohort study. Lancet Oncol 6: 279-286

O’Brien PC, Fleming TR (1979) A multiple testing procedure for clinical trials. Biometrics 35: 549-556

Rakitinat V, Vasilevskaya IA, O'Dwyer PJ (2003) Additive interaction of oxaliplatin and 17-allylamina-17-demethoxygaldanamycin in colon cancer cell lines results from inhibition of nuclear factor-kb signaling. Cancer Res 63: 8003-8005

Scartozzi M, Bearzi I, Berardi R, Mandolesi A, Fabris G, Cascinu S (2004) Epidermal growth factor receptor (EGFR) status in primary colorectal tumors does not correlate with EGFR expression in related metastatic sites: implications for treatment with EGFR-targeted monoclonal antibodies. J Clin Oncol 22(23): 4772-4778

Sclabas GM, Fujioka S, Schmidt C, Fan Z, Evans DB, Chiao PJ (2003) Restoring apoptosis in pancreatic carcinoma cells by targeting the nuclear factor-kB signalling pathway with the anti-epidermal growth factor antibody IMC-225. J Gastrointest Surg 7(1): 37-43

Van Schaeybroeck S, Karaiskou-McCaul A, Kelly D, Longley D, Galligan L, Van Cutsem E, Johnston P (2005) Epidermal growth factor receptor activity determines response of colorectal cancer cells to gefitinib alone and in combination with chemotherapy. Clin Cancer Res 11: 7480-7489

Voboril R, Hochwald SN, Li J, Brank A, Weberova J, Wessels F, Moldawer LL, Camp ER, MacKay SL (2004) Inhibition of NF-kB augments sensitivity to 5-fluorouracil/folinic acid on in colon cancer. J Surg Res 120(2): 178-188

Xu JM, Azzariti A, Colucci G, Paradiso A (2003) The effect of gefitinib (Iressa, ZD1839) in combination with oxaliplatin is schedule-dependent in colon cancer cell lines. Cancer Chemother Pharmacol 52: 442-448

Zampino MG, Magni E, Massacesi C, Zaniboni A, Martignetti A, Zorzino L, Lorizzo K, Santoro L, Boselli S, de Braud F (2007) First clinical experience of o rally active epidermal growth factor receptor inhibitor combined with simplified FOLFOX6 as first-line treatment for metastatic colorectal cancer. Cancer 110: $752-758$ 